\title{
Fragmentación de escala y precarización. Referentes de la estructuración urbana convencional
}

\author{
Fragmentation of a precarization scale. \\ References to conventional urban structuring
}

Rafael Monroy

\begin{abstract}
Resumen
Los países subdesarrollados cumplen el papel de aprovisionamiento de factores de producción en la racionalidad económica contemporánea. Las condiciones territoriales y sociales locales refieren un proceso de fragmentación de escala y precarización porque existe una tendencia a la ocupación irrestricta del suelo para el emplazamiento económico y urbano, así como una extracción de recursos intensiva y un deterioro de la capacidad de reproducción social. En el caso mexicano, la pérdida de suelo agrícola implica la reducción de la producción de alimentos básicos para la población, particularmente el maíz, pero de la misma forma, la sustitución de selva y bosque están asociados a las condiciones de sobreexplotación de acuíferos en la región con la mayor aglomeración. Además, los registros de precariedad en la vivienda indican que poco más del $60 \%$ de la población se encuentra en tales condiciones, lo cual no valida la adopción de estrategias de competitividad urbana, por ejemplo.
\end{abstract}

Palabras clave: fragmentación territorial; precarización; desarrollo regional.

\begin{abstract}
Developing countries play the role of supplying the contemporary economy. The territorial and social conditions of the place refer to the scale fragmentation process and to the increase in the poor population because there is a tendency of unrestricted use of land in order to convert it into an urban and productive place. In the Mexican case, the loss of land used for agricultural purposes implies a reduction in basic food production for the population, particularly corn. Furthermore, the substitution of forests and woodlands is associated with the overexploitation of aquifers in the country's most populated region. In addition, the records of low housing conditions indicate that a little more than $60 \%$ of the population are in this situation, which does not justify the adoption of such strategies.
\end{abstract}

Keywords: territorial fragmentation; precarization; regional development. 


\section{Introducción}

La operación de la racionalidad económica contemporánea está direccionada al aprovechamiento de regiones donde se proveen condiciones preferenciales de instalación, costos menores de mano de obra, oferta de una diversidad de recursos naturales e incluso, certeza política para el emplazamiento. La competencia por los factores de producción deriva en una configuración territorial fragmentada y para el caso latinoamericano con un origen no occidental, en impactos a las formas de aprovechamiento ambiental socialmente determinadas, tanto en áreas con rasgos no convencionales y heterogéneos como en sectores urbanos, los cuales son susceptibles de integrarse a un proceso supra local que les sustituye o les precariza progresivamente.

El orden económico supra local ejerce una fuerza de fragmentación y precarización, característicos en los países subdesarrollados; tal vez los casos más representativos de ello son la reducción de la capacidad de reproducción social en las áreas con mayor concentración población; la pérdida de interrelaciones socioambientales tradicionales a costa de la imposición de esquemas de extracción de especies utilizadas en la obtención de compuestos activos para farmacéuticas; la sobreexplotación de recursos como agua y madera para transnacionales; la exploración de metales preciosos para el mercado de plata y oro, entre otros.

Como resultado de la inserción al proceso de reproducción económica global los rasgos heterogéneos y no convencionales del territorio son afectados por los patrones de reestructuración en los que se emplazan actividades económicas y se ejerce un aprovechamiento ambiental extensivo. Convencionalmente se considera que la adopción de estrategias de desarrollo económico es útil para redistribuir beneficios a los diferentes sectores sociales (Polese, 1994, p. 165), (Camagni, 2005, p. 230), aunque en la práctica estos demuestran patrones de desarrollo desigual, dispar (Pradilla, 2008, p. 276) o parcializado (Hiernaux y Torres, 2008, p. 126), donde en realidad se refiere un estado de polarización o de articulación diferenciada al proceso global de producción.

Los procesos territoriales observados en Latinoamérica demuestran consistentemente una fragmentación de escala del espacio, así como a la globalización de la precariedad en la sociedad. Tales dimensiones cobran significado en la medida que permiten describir el grado de desarrollo regional ocasionado por la inserción al proceso de producción global; en última instancia, dicho desarrollo se sustenta en la desarticulación de las formas de aprovechamiento ambiental socialmente determinadas, así como en la afectación de la capacidad de reproducción social misma. Ambas subyacen y resultan contradictorias a los patrones predominantes de desarrollo, obligando al replanteamiento de lo urbano desde una perspectiva multidimensional de lo territorial.

En realidad, los patrones de inserción mexicana a la economía global han funcionado como factores regionales desestructurantes (Eckstein, 1999, p. 255), (Kozac, 2011, p. 22), en la medida en que estos se conforman con esquemas de consolidación urbana competitiva basada en la accesibilidad y los servicios, 
mientras que importantes extensiones de la ciudad y las regiones se caracterizan por un aprovisionamiento de vivienda progresivo, no consolidado o en condiciones fuera de los márgenes de la regularidad, afectando a una proporción importante de población. Existen revisiones de la reproducción del esquema urbano precario, utilizando indicadores generales de vivienda (Talavera, Morales y Muñoz, 2010, p. 39) o refiriendo la forma de apropiación del espacio para habitar (Padilla y Ribbeck, 2009, p. 32), (Connolly, 2008, p. 163); en el caso del Consejo Nacional de Evaluación de la Política de Desarrollo Social (Coneval) por ejemplo, se discute la multidimensionalidad de la pobreza integrando satisfactores y recursos, entre los que destacan aquellos asociados a las condiciones materiales de vida (Boltvinik, 2010, p. 44).

El territorio también presenta un proceso de fragmentación extensivo que alcanza a sectores regionales no occidentalizados o poco urbanizados en donde la desarticulación del sector agrícola o su integración a acuerdos desiguales de intercambio regional, como el Tratado de Libre Comercio de América del Norte (TLCAN) (Gasca, 2003, p. 53) resultan en el abandono del sector y en el incremento de esquemas urbanos precarios; al respecto, las estrategias de flexibilización política y económica son centrales para reproducir extensivamente las condiciones inmediatas de vida de la población más adversas (Tello, 2010, p. 305), (Garza, 2011, p. 82).

Por tanto, la estructuración económica ha consolidado patrones territoriales de aglomeración, de forma tal que la proporción de población urbana se ha incrementado a casi tres cuartas partes del total nacional, alcanzando 56 zonas metropolitanas (Secretaría de Desarrollo Social, Consejo Nacional de Población, Instituto Nacional de Estadística, Geografía e Informática, 2007, p. 31). No obstante, el sistema urbano nacional entraña un crecimiento urbano caracterizado por tener signos de precariedad en uno de cada tres habitantes (Bournazou, 2008, p. 405), (Damian y Boltvinik, 2003, p. 55); dicha condición es contradictoria económicamente, debido a que la urbanización es considerada convencionalmente como proveedor de ventajas, dadas las economías de escala, el desarrollo científico-tecnológico, así como una amplia disponibilidad de equipamientos y servicios; incluso la contribución urbana al PIB total mundial es del orden del $50 \%$ (Organisation for Economic Co-operation and Development, 2006, p. 305) y alcanza $80 \%$ en los países más urbanizados de Latinoamérica y Europa (United Nations Human Settlements Programme, 2005, p. 17).

Por el contrario y a la luz de los indicadores de pobreza o indigencia prevalecientes, es posible discutir la hipótesis de que la urbanización o los procesos regionales de reestructuración territorial, solamente tienen efectos favorables en el desarrollo en la población como una consecuencia directa de su adopción o de la inserción misma al proceso global de producción. En primera instancia, el sistema urbano entraña una transformación cualitativa, pero esta no asegura una distribución homogénea de condiciones de vida relativamente estables, ni tampoco puede ser considerado un signo suficiente para mitigar desequilibrios emergentes como los crecientes costos derivados del cambio climático (Comisión Económica para América Latina, 
2010 , p. 19) y la afectación a los sectores menos occidentalizados.

La reproducción de esquemas urbanos precarios también puede ser considerada un signo vulnerabilidad regional en la medida que genera costos de producción y afecta los mecanismos de inserción al proceso económico global. Muestra de ello son las crecientes deseconomías de escala, así como los gastos de restauración o mitigación ambiental que en conjunto ponen en evidencia la fragilidad económica de la ciudad y de los patrones territoriales. Esta condición refleja una condición de fragilidad del estado para asumir la regulación de las actividades económicas y de la misma forma, para establecer políticas públicas de interés social, que en última instancia son afectadas por el redireccionamiento del presupuesto público hacia problemas emergentes.

El objeto final de este trabajo es analizar las condiciones de reestructuración territorial en México, considerándola un efecto explícito de la inserción regional al proceso global de producción. Los indicadores utilizados para dicho propósito son la fragmentación territorial y la precarización; en la primera se asume que el estado de conservación de las regiones naturales es central para el soporte y funcionamiento de las aglomeraciones humanas y cuyo referente más importante es la vulnerabilidad y la dependencia ambiental de la región con mayor aportación económica en México. Para determinar la escala de la fragmentación, se estima la tasa de sustitución de selva baja caducifolia y bosque entre 2000 y 2010, considerados estos tipos de vegetación los principales proveedores de agua para el sistema urbano; al mismo tiempo, se estima la tasa de sustitución del sector agrícola y la consecuente pérdida de productos de la canasta básica para los mexicanos. En lo que respecta a la precarización, se estima la distribución de los indicadores generales de la vivienda precaria en el territorio nacional, así como la proporción de población afectada por dicha condición; los indicadores convencionales adoptados para su descripción incluyen la disponibilidad de agua corriente, drenaje, así como la proporción de población habitando en viviendas no propias, con algún nivel de hacinamiento y con piso de tierra. La presunción general es que la adopción de medidas económicas para soportar el funcionamiento general del circuito del capital encuentra desequilibrios sistémicos que en el caso mexicano pueden evidenciarse con base en la fragmentación de escala y la precarización.

\section{Fragmentación de escala}

El capitalismo contemporáneo está condicionado por el aprovechamiento de factores de producción; las aglomeraciones humanas y la disponibilidad de recursos naturales cumplen un papel central para dicho proceso e incluso representan la posibilidad misma de reproducción del sistema. El discurso occidentalizante subraya la relevancia económica de las aglomeraciones humanas en términos de su capacidad productiva, los indicadores convencionales útiles para ello son el Producto Interno Bruto (PIB), la tasa de empleo y el desarrollo tecnológico, lo cual justifica la expansión urbana en países subdesarrollados. El caso mexicano no es la 
excepción, dado que el Índice de Desarrollo Humano (IDH) es alto y consistente con una propuesta de política pública de expansión urbana; dicha estimación es considerada similar en economías Europeas y de América del Norte, como Islandia, Noruega o Canadá; (Programa de Naciones Unidas para el Desarrollo, 2009, p. 231) e incluso, el PIB nacional y per cápita también califican al país como la décima cuarta economía del planeta (The World Bank, 2009, p. 199), ver Cuadro 1.

Por tanto, en la discusión de lo urbano prevalece la idea de que esta es una condición sine quanon para transformar las condiciones de vida de la sociedad. Considerando la apreciación del IDH, es posible subrayar que se trata de una perspectiva analítica con criterios de evaluación homogéneos para todos los países a pesar de las disparidades regionales y de los impactos en las regiones subdesarrolladas que son producto de la urbanización. Dichos impactos incluyen la fragmentación de escala asociada al aprovechamiento intensivo de recursos locales, pero también entraña la desestructuración de la fuerza de trabajo, sometida a criterios de control y administración con estricto apego al funcionamiento del circuito global del capital. Es un hecho que la región latinoamericana ha demostrado consistentemente una serie

Cuadro 1 - Producto Interno Bruto e Índice de Desarrollo Humano

\begin{tabular}{l|c|c|c|c}
\hline \multirow{2}{*}{ País } & \multicolumn{2}{|c|}{ PIB } & \multicolumn{2}{c}{ IDH } \\
\cline { 2 - 5 } & $\begin{array}{c}\text { Miles de Millones } \\
\text { dólares }\end{array}$ & $\begin{array}{c}\text { Per cápita } \\
\text { miles de dólares }\end{array}$ & 2008 & 2009 \\
\hline Estado Unidos & 13,811 & 45 & Alto & Alto \\
Japón & 4,376 & 33 & Alto & Alto \\
Alemania & 3,297 & 34 & Alto & Alto \\
China & 3,280 & 5 & Medio & Medio \\
Reino Unido & 2,727 & 35 & Alto & Alto \\
Francia & 2,562 & 33 & Alto & Alto \\
Italia & 2,107 & 30 & Alto & Alto \\
España & 1,429 & 31 & Alto & Alto \\
Canadá & 1,326 & 35 & Alto & Alto \\
Brasil & 1,314 & 9 & Alto & Alto \\
Rusia & 1,291 & 14 & Alto & Alto \\
India & 1,170 & 2 & Medio & Medio \\
Corea & 969 & 24 & Alto & Alto \\
México & 893 & 14 & Alto & Alto \\
\hline
\end{tabular}

Fonte: Elaboración propia basada en The world bank, (2009); Programa de Naciones Unidas para el Desarrollo (2007); Programa de Naciones Unidas para el Desarrollo (2009). 
de evidencias que indican una estructuración económica del territorio con amplias consecuencias negativas y patrones de desarrollo desigual (Veiga, 2009, p. 52), (Poggiese, 2004, p. 231), (Aguilar, 2006, p. 6) 0 en su defecto, con una creciente necesidad de reequilibrar los costos sociales e incluso ambientales (Galindo, 2009, p. 54).

En el criterio convencional se asocian los niveles de urbanización con el desarrollo humano o en su defecto, los costos sociales y ambientales de la ciudad se asumen como deseconomías de aglomeración o externalidades negativas, desacreditando su origen en la expansión urbana. La urbanización subdesarrollada registra una creciente tasa de fragmentación territorial que afecta directamente la capacidad productiva de las regiones o por el contrario, resulta en una distribución diferencial de las condiciones económicas.

\section{Sustitución de selva baja caducifolia y bosque}

La fragmentación territorial está asociada a la racionalidad urbana y refleja una incorporación parcial al proceso global de producción (Kozac, 2011, p. 22); se caracteriza por la eliminación o sustitución progresiva de áreas naturales homogéneas, cuyos impactos significan la pérdida de funciones y servicios ambientales necesarios para la sociedad e incluyen la diversidad, la regulación energética, la provisión de agua u oxígeno, entre los más importantes (Constanza et al., 1997), (Gómez y De Groot, 2007).
El sistema urbano en México ocupa aproximadamente $1.1 \%$ del territorio nacional, proporción que es cuatro veces mayor a la de hace dos décadas (Martínez y Monroy-Ortiz, 2010, p. 11). La tasa de expansión urbana refleja una mayor fragmentación de escala en el país; de hecho, la mayor aglomeración urbana del país y también la más productiva económicamente, se localiza en el eje volcánico transversal entre los $18^{\circ}$ y $22^{\circ}$ de latitud norte, incluyendo 15 estados ( Guanajuato, Hidalgo, Querétaro, San Luís Potosí, Jalisco, Colima, México, Michoacán, Morelos, Puebla, Tláxcala, Distrito Federal, Estado de México, Veracruz), como se aprecia en el Mapa 1 (MonroyOrtiz, R. y Monroy, R., 2007), llevando a una desestructuración ambiental de mayor orden.

Dicha desestructuración puede estimarse en dos sentidos: con la fragmentación de la mayor reserva de bosque y selva baja caducifolia del país, ver Mapa 2, la cual lleva a la pérdida de resiliencia o de la capacidad mitigación de las eventualidades ambientales es decir, la falta de aprovisionamiento de servicios fundamentales como el agua o la regulación de la contaminación, en los cuales se cifra la capacidad de reproducción social o la mitigación del deterioro de las condiciones de vida de la población. Además, los grupos sociales con formas de desarrollo no occidental son los más afectados. En general, la resiliencia y la provisión de alimentos soportan una de las argumentaciones más importantes para replantear los patrones regionales de reestructuración territorial, dado que éstos implican una seria amenaza para el funcionamiento de las aglomeraciones humanas. 
Mapa 1 - Concentración urbana en México ( $18^{\circ}$ y $22^{\circ}$ de latitud norte)

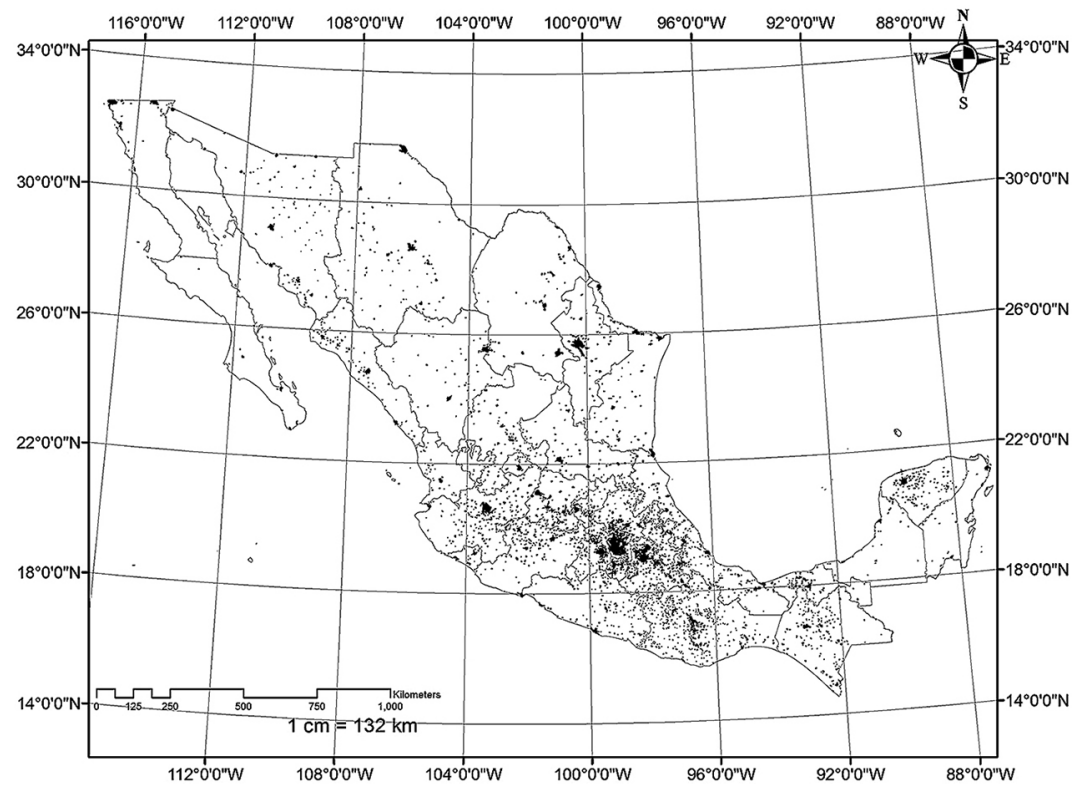

Fuente: Elaboración propia basada en Instituto Nacional de Estadística, Geografía e Informática (2005).

Mapa 2 - Urbanización sobre reservas de bosque y selva baja.

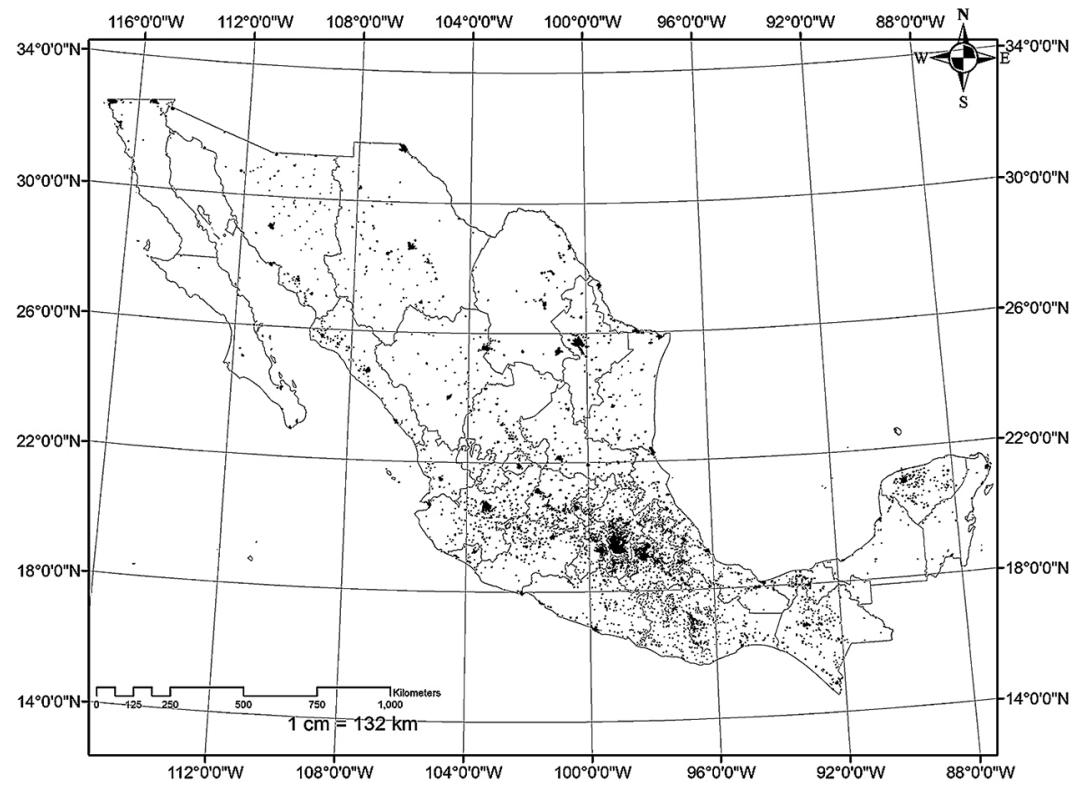

Fuente: Elaboración propia con base en Comisión Nacional para el Conocimiento y Uso de la Biodiversidad - Conabio (1999). "Uso de suelo y vegetación modificado por Conabio". Escala 1: 1000 000. Comisión Nacional para el Conocimiento y Uso de la Biodiversidad. Ciudad de México, México. Shape manejado en ARC GIS 9.2, tomado de Metadatos y mapoteca digital, Conabio digital. 
Por tanto, los efectos de la fragmentación territorial enlazan dos grandes condiciones: por una parte, la tasa de crecimiento urbano identificada según los tipos de vegetación que contienen la mayor cantidad de especies del país,

Entre 1990 y 2010, los cuatro tipos de uso del suelo con mayor tasa de sustitución fueron el agrícola de temporal (42.75\%), el agrícola de riego (26.58\%), el pastizal cultivado $(10.85 \%)$, la selva baja caducifolia (5.24\%) y el bosque (4.69\%). (Monroy-Ortiz, 2013, p. 169)

En segunda instancia, la pérdida de diversidad implica que $60 \%$ de la región más urbanizada presenta una decreciente disponibilidad de agua, estimándose en una condición de sobreexplotación de los acuíferos, por ejemplo (Comisión Nacional del Agua, 2010), lo cual representa un serio riesgo para la región que concentra a 40 millones de habitantes y a las actividades económicas que generan $60.29 \%$ del PIB nacional.

\section{Sustitución de área agrícola}

La desarticulación del sector agrícola también es resultado de la fragmentación territorial; el impacto más importante tiene que ver con la pérdida progresiva de la autosuficiencia alimentaria. En este sentido, existen estimaciones de la pérdida de superficie cultivada, la cual afecta particularmente al maíz que es un grano básico e incluso eje rector de la dieta de los mexicanos. De hecho, alrededor $16 \%$ del territorio nacional es suelo agrícola, el cual ocupa aproximadamente $310,778.98 \mathrm{~km}^{2} ; 69 \%$ agricultura de temporal en una proporción de dos a uno respecto a la de riego. Si bien el sector agrícola significa 4.5\% del Producto Interno Bruto y ocupa 18\% de la Población Económicamente Activa (PEA), el principal resultado de la desarticulación de dicho sector implica entre otras cosas, el riesgo de perder el abastecimiento y la disponibilidad de alimentos, más aún cuando se estima que alrededor de 18.6 millones en se encuentran en situación de pobreza alimentaria (Consejo Nacional de Evaluación de la Política de Desarrollo Social, 2010, p. 22).

En este sentido, la pérdida de superficie cultivada también lleva a la importación de granos básicos o en general de alimentos para la población, así como a un incremento en el costo para su adquisición. El sector urbano que multiplica la demanda de alimentos resulta particularmente rentable para el capital, pero también entraña crecientes costos sociales y en todo caso, representa un proceso de desarticulación o fragmentación que no se encuentra en la discusión de la teoría urbana occidental. La localización e incluso el emplazamiento de sectores económicos en las ciudades sobresalen como esquemas de reconocimiento y adecuación de la política pública a pesar de que se registra una pérdida progresiva de la superficie cultivada. Al respecto, se estima que la expansión urbana es responsable de la sustitución de suelo agrícola; para la última década se perdieron alrededor de $6,000 \mathrm{~km}^{2}$ de agricultura de temporal y $4,000 \mathrm{~km}^{2}$ de agricultura de riego, siendo el suelo agrícola de temporal el más afectado. La penetración del sector urbano en suelo agrícola demuestra una afectación similar en términos de localización alrededor de los $18^{\circ}$ y $22^{\circ}$ de latitud norte, como se aprecia en el Mapa 3. 
Mapa 3 - Expansión urbana sobre suelo agrícola (riego y temporal)

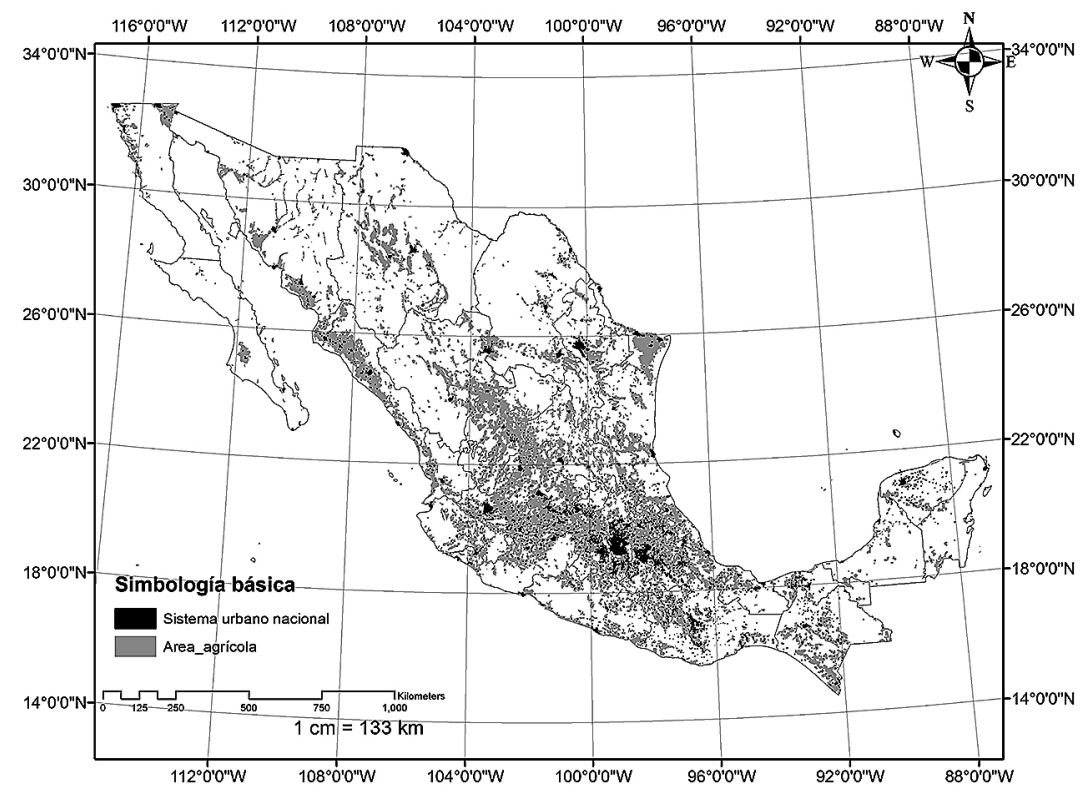

Fuente: Elaboración propia con base en Comisión Nacional para el Conocimiento y Uso de la Biodiversidad - Conabio (1999). "Uso de suelo y vegetación modificado por Conabio". Escala 1: 1000 000. Comisión Nacional para el Conocimiento y Uso de la Biodiversidad. Ciudad de México, México. Shape manejado en ARC GIS 9.2, tomado de Metadatos y mapoteca digital, Conabio digital.

Regionalmente el centro occidente del país registra la mayor pérdida de suelo agrícola; tanto los beneficios económicos directos 0 derivados de su aprovechamiento como la capacidad productiva del país son afectados. En este sentido, los estados más afectados son México, Morelos, Michoacan, Jalisco y Colima, mientras que en un segundo rango sobresalen
Puebla, Distrito Federal, Guanajuato, Coahuila, Sinaloa, Sonora y Baja California Sur, como se aprecia en el Cuadro 2. Resalta que el producto mayormente afectado es el maíz, por lo que al ser un producto indispensable en la dieta del mexicano, puede propiciar efectos altamente negativos para la población en el mediano y largo plazo. 
Cuadro 2 - Productos agrícolas afectados por la expansión urbana

\begin{tabular}{|c|c|c|c|}
\hline \multicolumn{4}{|c|}{ Intensidad de expansión urbana alta } \\
\hline \multirow[t]{2}{*}{ Estados } & \multirow{2}{*}{$\begin{array}{l}\text { Reporte alimentario } \\
\quad \text { (Inegi, 2008) }\end{array}$} & \multicolumn{2}{|c|}{$\begin{array}{l}\text { Anuario estadístico de la producción agropecuaria } \\
\text { (Sagarpa, 2006) }\end{array}$} \\
\hline & & Superficie sembrada & Valor de producción \\
\hline Baja California & Jitomate & Trigo grano & Tomate rojo \\
\hline Colima & Limón & Pastos & Limón \\
\hline Estado de México & Maíz & Maíz & Maíz \\
\hline Michoacán & Aguacate & Maíz & Maíz \\
\hline Morelos & Caña de azúcar & Maíz & Caña de azúcar \\
\hline Jalisco & Maíz & Maíz & Agave \\
\hline \multicolumn{4}{|c|}{ Intensidad de expansión urbana media alta } \\
\hline Baja California Sur & Jitomate & Garbanzo blanco & Chile verde \\
\hline Coahuila & Рapa & Zacate & Papa \\
\hline Distrito Federal & Papa & Avena forrajera & Nopal \\
\hline Guanajuato & Sorgo & Maíz & Sorgo grano \\
\hline Puebla & Maíz & Maíz & Maíz \\
\hline Sonora & Uva & Trigo & Trigo grano \\
\hline Sinaloa & Maíz & Maíz & Maíz \\
\hline
\end{tabular}

Fuente: Martínez y Monroy-Ortiz (2010).

La fragmentación de escala demuestra la consolidación del sector urbano, debido a la adopción de esquemas homogéneos de reestructuración territorial; a pesar de las múltiples deseconomias, la posición teórica occidental los promueve. La contrastación de dos conceptualizaciones respecto al sector urbano, permite aproximarse o estimar un estado crítico de subsistencia; el ejemplo más claro es la pérdida de superficie cultivada y al mismo tiempo, la creciente dependencia alimentaria del país. Igualmente importante, la sustitución de suelo forestal o de selva entraña la pérdida de disponibilidad de agua para la región con mayor crecimiento urbano.
La fragmentación de escala es una medida de la adopción de esquemas occidentales de desarrollo, para los que no existe una capacidad de carga suficiente en términos del tiempo de reproducción, ni tampoco para soportar plazo alguno en el futuro. La perspectiva más equilibrada para reproducir la aglomeración humana contemporánea, tiene que ver con la consideración de tales restricciones; en caso contrario, los costos de un esquema demandante y acumulativo se incrementarán insosteniblemente para los países subdesarrollados, ocasionando una dependencia mayor a la que se observa ya en dichas sociedades. 


\section{Precarización}

La globalización se cierne sobre el subdesarrollo como un patrón de intercambio o interrelación dispar en donde la oferta de mano de obra, la desregulación para su aprovechamiento, así como la múltiple extensión de zonas ambientales relativamente homogéneas son objeto de dicho intercambio. Por el contrario, el papel de la administración e incluso del consumo en el circuito global de producción, se lleva a cabo en el mundo desarrollado. En realidad, la población local no participa de los supuestos beneficios del libre mercado, por el contrario, es objeto de transformación, adaptación e incluso de exigencia para su correcta instalación en un esquema internacional de intercambio en el que no corresponde sino un papel de aprovisionamiento de mano de obra y recursos.

En realidad, la globalización de la precariedad permite subrayar los desequilibrios derivados del intercambio desigual de desarrollo-subdesarrollo. Entre los principales elementos para tal diagnostico se encuentran las formas de evaluación de las condiciones de vida de la población; éstos incluyen el nivel de ingreso, las necesidades básicas insatisfechas e incluso, algunas descripciones como la marginación o la segregación (Hernández Laos, 2006; Boltvinik, 2010). El asunto entonces tiene que ver con la reproducción estructural de una disparidad o de condiciones desiguales de integración económica; mientras el mayor consumo energético se da en los países del norte, el sur es fuente primaria del combustible fósil o por el contrario, la mayor oferta de los países subdesarrollados es la mano de obra mal remunerada y los crecientes ejércitos de reserva, supeditados al proceso de instalación del capital.

Tan solo en términos del nivel de ingreso, México demuestra una alta concentración de población económicamente activa con una percepción por debajo de los salarios mínimos indispensables para su reproducción social. Se estima que $60 \%$ de la población se encuentra en dicho supuesto y al mismo tiempo, alrededor del $82 \%$ de los municipios del país demuestran consistentemente dicha configuración. En realidad, la adopción de intercambios desiguales con base en Inversión Extranjera Directa o el recibimiento flexibilizado de Empresas Transnacionales resulta un esquema que poco favorece al entorno social o a las condiciones ambientales mismas; al respecto, existe un registro de desequilibrios territoriales o de afectados ambientales ocasionados por la instalación de capital extranjero.

En el sentido convencional, la población con algún rasgo desestructurante resulta un ejemplo directo de los patrones de intercambio desigual, pero de la misma forma, expresa una forma de integración que entraña efectos adversos. La revisión de dichos efectos es posible desde diferentes enfoques; en todo caso, afines o no afines a la racionalidad económica o como consecuencia directa del tratamiento diferencial en la integración regional. Sin embargo, la lógica de las políticas públicas enfocadas al territorio implica directamente una expansión urbana que favorezca el mejoramiento de la calidad de vida de la sociedad; la evaluación convencional de ello se soporta en el nivel de ingreso mismo o por el contrario, en términos de la creciente capacidad urbana para proveer servicios para la población. 
Debido a ello, una revisión de los patrones de precariedad es útil para demostrar los desequilibrios estructurales derivados de la forma prevaleciente de intercambio global, mismos que configuran una organización de subsistencia o un arreglo forzado en las aglomeraciones humanas como formas del urbanismo contemporáneo y donde quedan en evidencia los intercambios irrestrictos a los que están sometidas todas las regiones del planeta; es decir, se demuestran los efectos de un movimiento extensivo y extraterritorial causantes de serias transformaciones sociales.

En términos convencionales, los desequilibrios pueden asumir diferentes formas de análisis como las que se mencionan antes sin embargo, la discusión urbana refiere cambios directos en las condiciones de vida de la población como un resultado directo de la urbanización; en esta medida, el incremento progresivo de la precariedad es determinante de patrones poco homogéneos de desarrollo. Por tanto, los asentamientos precarios resultan centrales para contra argumentar dado que implican una serie de condiciones de vida asociadas a la vivienda, incluyendo: materiales resistentes, área habitable mínima, acceso a agua, acceso a drenaje y seguridad en la propiedad (United Nations Human Settlements Programme, 2006, p. 19). Es decir, para que un asentamiento sea considerado como precario debe registrar al menos uno de dichos indicadores, en cuyo caso implican dificultades para la vida humana en términos económicos, de salud y protección. En este marco, la reproducción de esquemas precarios y su respectiva distribución en los asentamientos humanos explican la disparidad o en todo caso, la particularizan territorialmente. La hipótesis de la localización económica o en su defecto, de la geografía económica implica el mejoramiento de las capacidades de la población para adaptarse al entorno social regulado bajo criterios de mercado. La distribución diferenciada de los beneficios económicos expresa una disfuncionalidad particularizada en los asentamientos precarios, incluyendo su localización y distribución. Debido a ello, la cantidad de municipios, vivienda y población identificadas bajo algún indicador de precariedad demuestran el desarrollo diferenciado, el cual es una evidencia identificada, pero no reconocida de las formas contemporáneas de gestionar el desarrollo urbano.

\section{Características de la precariedad en México}

El parque habitacional en el país se estima en poco más de 30 millones de viviendas; agrupadas en propias, no propias, particulares o colectivas (Instituto Nacional de Estadística y Geografía, 2005, p. 956). Con base en este universo es posible determinar la prevalencia precariedad por municipio, dando una identificación territorial a los procesos de globalización; es decir, se territorializan los desequilibrios asociados a un proceso de urbanización o a una forma de adoptar el emplazamiento económico. Como se comenta antes, se asume que la urbanización permite proveer beneficios a una mayor cantidad de población; la precariedad por el contrario, demuestra una inconsistencia en tal supuesto. Prácticamente, la sociedad más urbana registra más desequilibrios en el sentido de 
las condiciones inmediatas de vida, cuando la presunción de la política pública es opuesta.

Derivado de la revisión de las condiciones de precariedad en la vivienda se encuentran las siguientes generalidades: a) la cobertura de agua y drenaje representan el servicio mejor distribuido, dado que la mayor parte de los municipios se clasifica de media a alta respecto al parque habitacional; b) las viviendas con piso de tierra alcanzan $40 \%$ del total registrado en 1662 municipios, es decir 4 de cada 10; c) en 2117 municipios, es decir alrededor de $86 \%$ del total nacional, se estima que 4 de cada 10 viviendas registra algún nivel de hacinamiento; d) en 2054 municipios la cantidad de viviendas con problemas de propiedad se calcula entre 10 a $40 \%$ del total habitacional, ver Gráfica 1.

\section{Gráfica 1 - Número de municipios, según rango de predominancia de los determinantes de precariedad}

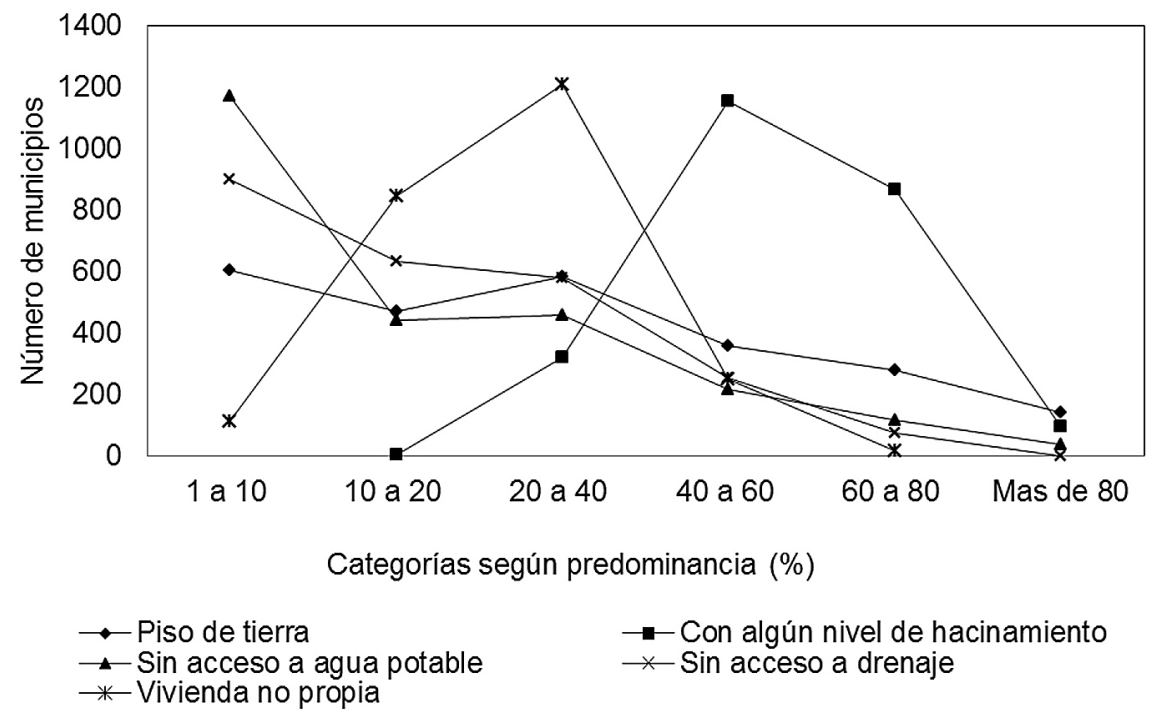

Fuente: Elaboración propia basada en Instituto Nacional de Estadística y Geografía (2000), XII Censo general de población y vivienda. Instituto Nacional de Estadística y Geografía (2005), II Conteo de población y vivienda. 
Considerando la descripción convencional de la precariedad, resulta particularmente grave que 4 de cada 10 viviendas expresen algún nivel de hacinamiento o en su defecto, que la población no sea propietaria de la vivienda. En primera instancia, la distribución de beneficios o el mejoramiento de calidad de vida de la población se válida marginalmente; pero aún, la distribución del hacinamiento 0 de la población que no es propietaria de su vivienda resulta predominante en poco más del $80 \%$ de los municipios. Tales indicadores reflejan un desequilibrio que poco se precisa en la política pública sustentada en criterios de localización o geografía económica; no se ve reflejada en estrategias de orden común que atiendan tales disparidades.

En este sentido, existe un reconocimiento oficial del déficit de vivienda o de las condiciones precarias de la población, sin embargo el objetivo de cubrir la demanda real en el país encuentra serias diferencias con la apertura para el capital inmobiliario. Por una parte se reconoce el déficit pero, por otra la política nacional Ileva a la flexibilización en la instalación del capital inmobiliario, bajo el supuesto de atender dicho déficit; el resultado es un incremento del indicador y en todo caso, la reducción del espacio útil para otros propósitos, entre los que desataca precisamente la producción de alimentos o la provisión misma de servicios ambientales, como el agua o la captura de carbono. La apertura y flexibilización del suelo urbanizable no garantiza la reducción déficit en el sector, dada la oferta para un nivel de ingreso predominantemente bajo o con baja capacidad para ser sujeto de crédito. En la práctica, la estimación oficial respecto al déficit de vivienda refiere una demanda de 4 279735 de unidades (Comisión Nacional de Fomento a la Vivienda, 2000, p. 32) y proyecta un incremento anual de aproximadamente 650.000 viviendas (Comisión Nacional de Fomento a la Vivienda, 2008, p. 11). La oferta del sector revisada desde las políticas públicas encuentra programas de gobierno como mi casa en donde la intención es proveer vivienda de interés social para la población, así como constructoras o desarrolladoras para el mismo sector identificadas en el padrón de la Cámara Nacional de la Industria de la Construcción. En este contexto, la mayor parte de los desarrollos inmobiliarios se concentra en Baja California Norte, Quintana Roo, Jalisco, Estado de México, Puebla y Veracruz, alcanzando una cantidad de proyectos entre 50 y 300 y con criterios similares en términos de dimensiones y costos, por ejemplo. No obstante, la posibilidad de acceder a un crédito para adquirir alguna vivienda es menor a nivel nacional.

En este orden de ideas, la precarización refleja una forma de aprovechamiento del capital inmobiliario con base en el desarrollo del sector habitacional; se consolida bajo el criterio de resolver el déficit de vivienda, pero en esencia se consiguen una cantidad importante de conjuntos habitacionales para los que no existe un mercado accesible para la población mexicana. Por ello, existen registros de conjuntos inmobiliarios poco ocupados 0 con bajos niveles de venta.

Por tanto, la atención a la demanda efectiva de vivienda tiene una serie de problemas estructurales que incluyen: la precaria distribución del ingreso porque pone de manifiesto la incapacidad económica de la población para adquirir vivienda y por otro 
lado, el Estado refleja una limitada capacidad para proveerla. En este sentido, se puede comentar que solamente 5 de 14 millones de trabajadores cotizando al Instituto del Fondo Nacional de la Vivienda para los Trabajadores (Infonavit) han sido atendidos en 37 años (iniciativa que reforma diversas disposiciones de la Ley de Instituto del Fondo Nacional de la Vivienda para los Trabajadores, 2009), Io cual lleva a un incremento acumulativo de la demanda, toda vez que las opciones privadas tienen pocas alternativas crediticias para los niveles de ingreso observados en la población.

En resumen, alrededor de $37.05 \%$ del total de viviendas en el país tiene algún nivel de hacinamiento; proporción que resulta equivalente a aproximadamente 4 veces la prevista por el estado, es decir 4 de cada 10 viviendas. Por otra parte, los problemas de propiedad se registran en $18.54 \%$ del parque habitacional. Atendiendo a tales proporciones se estima que 45 millones de personas viven con en algún grado de hacinamiento $\mathrm{y}$ alrededor de 22 millones, no cuentan con una vivienda propia, mientras que 13 millones habitan en una vivienda cuyo piso es de tierra. El mejoramiento de la calidad de vida descrito en el orden urbano como una estrategia de desarrollo, puede ser discutido en términos de la cantidad de población habitando en tales circunstancias.

Atendiendo a la evidencia, se puede comentar que ninguna región está exenta de hacinamiento, incluyendo a las aglomeraciones urbanas con las mayores tasas de urbanización e incluso aquellas consideradas centrales para la capacidad económica del país. En este sentido, la urbanización concentra una importante capacidad productiva como se destaca en los criterios urbanísticos occidentales, sin embargo los costos sociales que resultan de la adopción ciertos patrones territoriales alcanzan una escala apremiante desde que cerca de la mitad de la población no ha modificado sus condiciones inmediatas de vida.

La distribución regional de la precariedad es un determinante de la política urbana, dada su escala en el territorio nacional; al mismo tiempo, se cierne como un objeto de estudio desde el sur en la perspectiva de construir un urbanismo no convencional o desoccidentalizado, es decir desvistiéndole de rasgos que no responden a un patrón regional de desarrollo urbano o que en su defecto, son causantes de serios desequilibrios locales.

Cabe destacar que una política pública desoccidentalizada debiera tener entre sus ejes de atención la distribución de la vivienda con algún nivel de hacinamiento, dado que ésta predomina en las categorías de medio a alto en el parque habitacional por municipio. $\mathrm{Si}$ bien se registra un promedio alto en todo el territorio nacional, la mayor concentración del hacinamiento se observa en el centro y sur, particularmente Guerrero, Oaxaca, Chiapas, Veracruz, y la península de Yucatán, como se aprecia en el Mapa 4. 
Mapa 4 - Vivienda con algún nivel de hacinamiento, según rango de incidencia

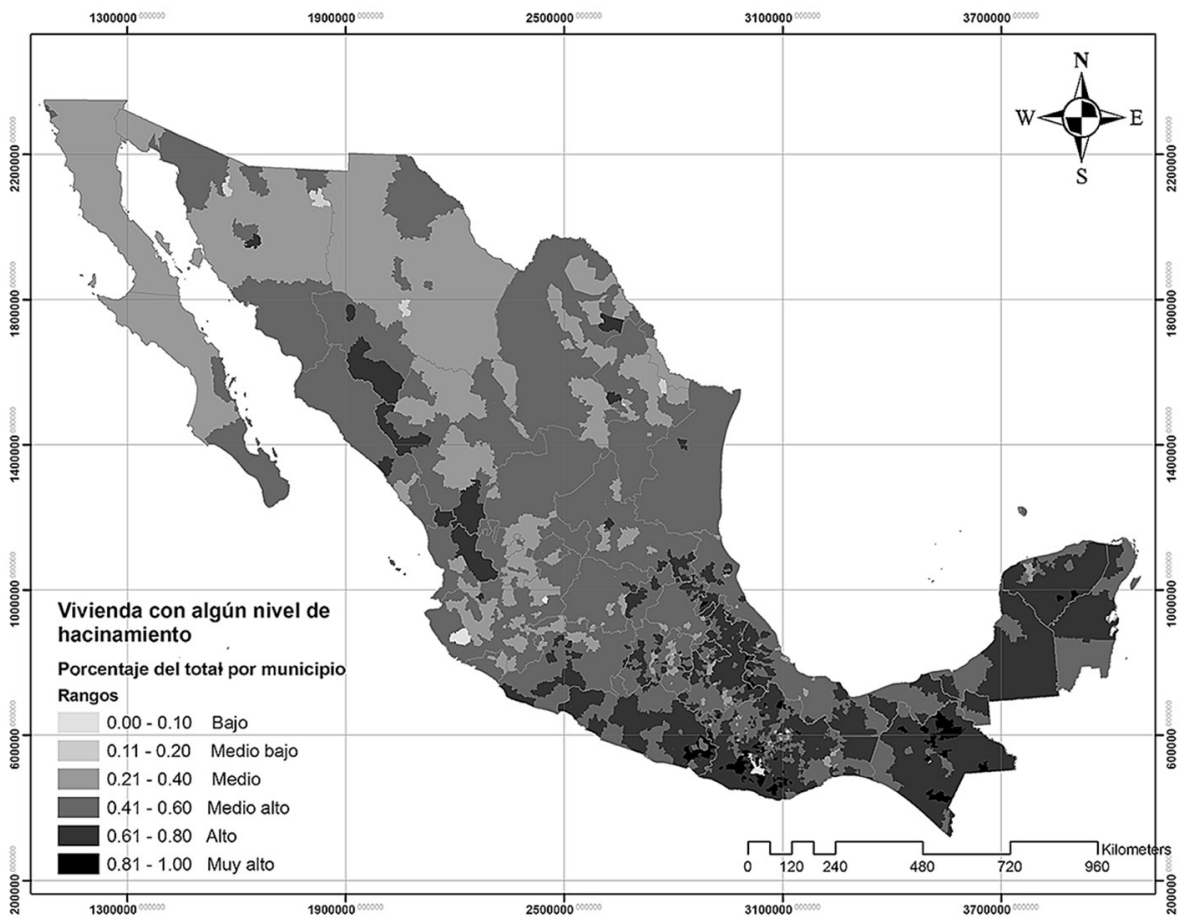

Fuente: Elaboración propia basada en Instituto Nacional de Estadística y Geografía (2009). [Metadatos y mapoteca digital]. Instituto Nacional de Estadística y Geografía (2000), XII Censo general de población y vivienda. Instituto Nacional de Estadística y Geografía (2005), II Conteo de población y vivienda.

En este orden de ideas, los problemas de propiedad de la vivienda se concentra en los rangos medio bajo y medio, los cuales alcanzan aproximadamente $40 \%$ del total de viviendas por municipio; las regiones por arriba de la media se localizan del centro al norte del país, como se observa en el Mapa 5.

La revisión general de la precarización tiene como propósito subrayar los efectos de la integración desigual a procesos de intercambio económico; se reconoce que el circuito del capital consigue emplazamientos por una serie de beneficios que éstos le proveen. De la misma forma, la política pública es útil como apoyo logístico para cristalizar dicho proceso de instalación; el sustento sin embargo, se concentra en la posibilidad obtener mayor desarrollo económico y en consecuencia, necesario para modificar las condiciones de vida de la población. La discusión al respecto incluye posicionamientos que justifican la localización y la distribución geográfica favorable para alcanzar la meta del desarrollo; se trata del urbanismo denominado aquí como occidentalizado y que prevalece en la política pública sin embargo, los impactos de dicha 
Mapa 5 - Vivienda no propia, según rango de incidencia

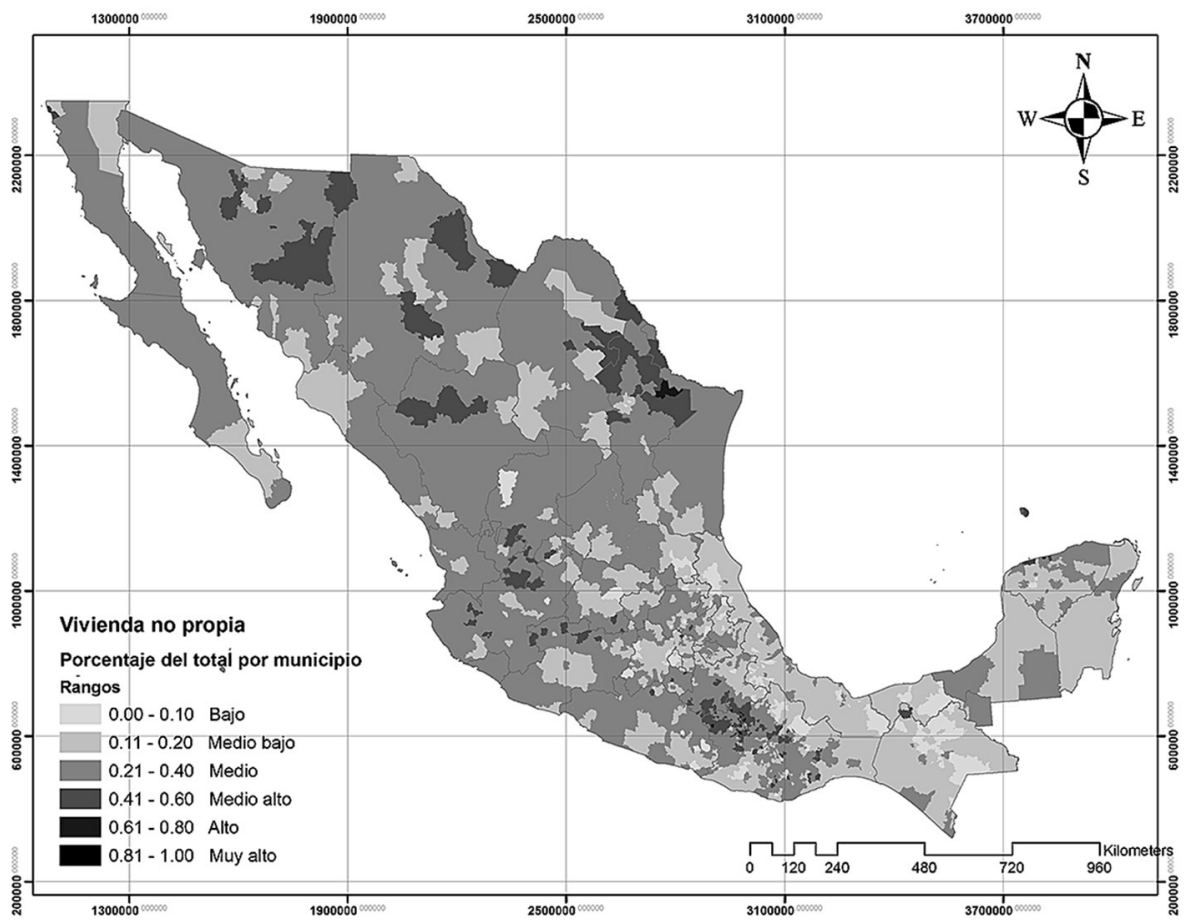

Fuente: Elaboración propia basada en Instituto Nacional de Estadística y Geografía (2009). [Metadatos y mapoteca digital]. Instituto Nacional de Estadística y Geografía (2000), XII Censo general de población y vivienda. Instituto Nacional de Estadística y Geografía (2005), II Conteo de población y vivienda.

adopción deja múltiples desequilibrios que han sido comentados en términos del hacinamiento y los problemas de propiedad. No es un propósito agotar la demostración empírica de los desequilibrios, sino de alguna forma utilizar los criterios cuantitativos para discutir aquellas posiciones convencionales que no modifican la perspectiva a pesar de encontrar tales niveles de deterioro social. En todo caso, predomina la visión de la capacidad económica o del desarrollo humano convencional por arriba de la creciente desarticulación de la sociedad urbana.

\section{Reflexiones para una discusión no convencional}

Las regiones atraviesan un proceso de interrelación no prevista o con particularidades poco preparadas para su intervención; las normas de interacción global son propuestas desde la esfera virtual del aprovechamiento de los factores de producción, sin que en éstos medie una capacidad de carga o unas condiciones sociales no occidentales como las que residen en el ámbito latinoamericano e 
incluyen formas de organización tradicionales o en todo caso, mixtas pero con componentes poco afines al contexto global.

La falta de previsión o las particularidades poco occidentalizadas son el marco de la intervención territorial local, a pesar de los emplazamientos económicos que favorecen su desarticulación. En este sentido, la mayor parte de la política pública e incluso de la discusión académica se han concentrado en ajustar los esquemas de intervención adquiridos, llevando a una flexibilización del suelo, pero aún, a una creciente desarticulación de las características inherentes al contexto latinoamericano. Los consecuentes desequilibrios demuestran una resistencia social organizada por afectados ambientales o afectados urbanos poco considerados en la definición del perfil urbanístico o territorial asumido. Al mismo tiempo, las disfuncionalidades de las decisiones administrativas no son objeto de evaluación crítica, ni mucho menos de revaloración horizontal e incluyente; en este sentido, resulta interesante como el discurso convencional adopta esquemas en el sentido de la horizontalidad y les llama gobernanza o participación social, sin que medie una decisión realmente de este tipo.

Por tanto, se observa una perspectiva analítica convencional con efectos poco favorables para la sociedad; al mismo tiempo, no se somete a juicio alguno los crecientes desequilibrios o en el mejor de los casos, se canalizan en una segunda versión de las estrategias asumidas inicialmente, bajo el criterio de que el desarrollo económico y los emplazamientos que lo permiten son precisamente la respuesta a los desequilibrios previamente generados.
En este documento se discuten dichos desequilibrios de una forma cuantitativa; la propuesta es abordar la fragmentación de escala asumiendo que las economías de escala o aglomeración implican dialécticamente un desequilibrio. La perspectiva para el país incluye datos reveladores de la disfuncionalidad de los mecanismos de intercambio global asumidos en el país. Estos incluyen la pérdida progresiva de la mayor reserva de bosque y selva, en ambos casos proveedores de servicios ambientales básicos como la disponibilidad de agua, la cual no es objeto de regulación o siquiera de intervención en el urbanismo occidentalizado. De la misma forma, la reducción progresiva de la superficie cultivada y de la pérdida de la autosuficiencia alimenticia, afectando particularmente al maíz que se identifica como fuente básica de la alimentación local. Tanto los servicios ambientales de disponibilidad de agua como de producción de alimentos, se refieren forzosamente a una perspectiva dentro del marxismo ecológico o de la economía de los recursos, sin embargo debieran formar parte de la opción del urbanismo no convencional para reestructurar las condiciones ambientales en franco riesgo y vulnerabilidad.

Por otro lado, la fragmentación de escala tiene una particularización social referida como globalización de la precariedad; se asumen en este caso que la sociedad no encuentra los supuestos beneficios en la calidad de vida. Por el contrario, los indicadores más elementales de las condiciones de vida demuestran consistentemente un deterioro estructural tan solo en términos del hacinamiento o de los problemas de propiedad. Incluso, se hace una revisión de las estrategias adoptadas para resolver el déficit de vivienda, toda vez 
que el desarrollo económico actúa bajo el supuesto de resolver dichas condiciones. La consecuencia inmediata es que el apoyo se vierte al sector inmobiliario en una suerte de articulación de problemas sociales y desarrollo económico, lo cual solo termina por consolidar al capital inmobiliario y a demostrar los graves problemas estructurales de la población para acceder a una vivienda; sin mencionar las particularidades de la vivienda.

En ambos casos, la base material necesaria para la reproducción social, así como las condiciones mismas de integración regional son parte de la adopción de un urbanismo occidentalizado; en todo caso, demuestran precisamente las inconsistencias de su operación. El discurso occidental asigna a la localización la geografía económica una capacidad que se demuestra cuantitativamente en términos del Índice de Desarrollo Humano o por la capacidad para contribuir al Producto Interno Bruto, de forma que las particularidades son sesgadas y no con una perspectiva diferencial. Debiera existir un Esquema No Occidental de Aprovechamiento Ambiental (ENOA), en donde se identificaran aspectos como la biodiversidad, las regiones menos fragmentadas, así como aquellas indígenas. Al mismo tiempo, el criterio urbanístico debiera asumir un esquema territorial determinado por el ENOA y no por el PIB. Por otro lado, la presunción del IDH recoge muy limitadamente rasgos locales; algunos ejemplos de uso común en las ciudades latinoamericanas debieran ser eje de análisis. Estos tienen que ver con el auto provisionamiento de alimentos en Huertos Frutícolas Tradicionales HFT, la venta de productos agrícolas locales, el intercambio de subsistencia, el aprovechamiento de plantas medicinales o la misma adopción de formas de cultivo tradicionales, todos ellos practicados en ámbitos urbanos, pero no reconocidos como rentables. Al respecto, debiera manejarse una Categoría Heterogénea de Subsistencia (CAHES) para sustituir al IDH.

Finalmente, el ámbito urbano latinoamericano está determinado por una heterogeneidad que incluye tanto el Esquema No Occidental de Aprovechamiento Ambiental como la Categoría Heterogénea de Subsistencia. La política urbanística, la discusión teórica no convencional debiera pasar por tales matices, para dar una certeza local y desoccidentalizada.

\section{Rafael Monroy}

Universidad Autónoma del Estado de Morelos, Facultad de Arquitectura. Cuernavaca/Morelos, México.

rafaelmoor@hotmail.com 


\section{Referencias}

AGUILAR, A. G. (coord.). (2006). Las grandes aglomeraciones y su periferia regional. Experiencias en Latinoamérica y España. Distrito Federal/México, Universidad Nacional Autónoma de México.

BOLTVINIK, J. (2010). "Principios de la medición multidimensional de la pobreza". In: BOLTVINIK, J.; CHAKRAVARTY, S. R.; FOSTER, J. E.; GORDON, D.; HERNÁNDEZ CID, R.; SOTO DE LA ROSA, H. e MORA, M. Medición multidimensional de la pobreza en México. México, El Colegio de México.

BOURNAZOU, E. (2008). “La segregación social del espacio y la dimensión territorial en los estudios de pobreza urbana". In: CORDERA, R.; RAMIREZ KURI, P. e ZICCARDI, A. Pobreza, desigualdad y exclusión social en la ciudad del siglo XXI. México, Siglo XXI.

CAMAGNI, R. (2005). Economía urbana. España, Antoni Bosch.

COMISIÓN ECONÓMICA PARA AMÉRICA LATINA (2010). Cambio climático. Una perspectiva regional. Santiago/Chile, Autor.

COMISIÓN NACIONAL DE FOMENTO A LA VIVIENDA (2000). Rezago habitacional. México, Autor.

(2008). Programa nacional de vivienda 2007-2012: hacia un desarrollo habitacional sustentable. México, Autor.

COMISIÓN NACIONAL DEL AGUA (2010). Estadísticas del agua en México. Conagua, México.

COMISIÓN NACIONAL PARA EL CONOCIMIENTO Y USO DE LA BIODIVERSIDAD - CONABIO (1999). Uso de suelo y vegetación modificado por Conabio. Escala 1: 1000000 . Ciudad de México, México. Shape manejado en ARC GIS 9.2, tomado de Metadatos y mapoteca digital, Conabio digital.

CONNOLLY, P. (2008). “Urbanizaciones irregulares como forma dominante de ciudad”. In: IRACHETA, A. X. e MEDINA, S. Irregularidad y suelo urbano. México, Secretaría de Desarrollo Social.

CONSEJO NACIONAL DE EVALUACIÓN DE LA POLÍTICA DE DESARROLLO SOCIAL (2010). Dimensiones de la seguridad alimentaria: evaluación estratégica de nutrición y abasto. México, Autor.

CONSTANZA, R.; D'ARGE, R.; DE GROOT, R.; FARBER, S.; GRASSO, M.; HANNOT, B.; LIMBURG, K.; NAEEM, O'NEILL, R.; PARUELO, J.; RASKIN, R.; SUTTON, P. e VAN DEN BELT, M. (1997). The value of the world's ecosystem services and natural capital. Nature, v. 387, pp. 253-260.

DAMIAN, A. e BOLTVINIK, J. (2003). Evolución y características de la pobreza en México. Comercio exterior, v. 6, n. 6 .

ECKSTEIN, S. (1999). El estado y la pobreza urbana en México. México, Siglo XXI.

GALINDO, L. M. (2009). La economía del cambio climático en México. México/Distrito Federal, Secretaría de Medio Ambiente y Recursos Naturales.

GARZA, G., (2010). "La ciudad como fuerza productiva: desarrollo económico y competitividad". In: SOBRINO, J. Competitividad urbana. Una perspectiva global y para México. México, El Colegio de México.

GASCA, J. (2003). "Políticas regionales de primera y segunda generación. Hacia una nueva propuesta para las regiones mexicanas". In: ACEVEDO, V. A. e NAVARRO, J. C. L. (coords.). Globalidad, desarrollo y región. México, Universidad Michoacana de San Nicolás de Hidalgo. 
GÓMEZ, E. e DE GROOT, R. (2007). Capital Natural y Funciones de los Ecosistemas: explorando las Bases Ecológicas de la Economía. Ecosistemas, v. 16, n. 3, pp. 4-14.

HERNÁNDEZ LAOS, E. (2006). Bienestar, pobreza y vulnerabilidad: nuevas estimaciones para México. Comercio exterior, n. 53, pp. 453-465.

HIERNAUX, D. e TORRES, R. (2008). "Desarrollo territorial en México: un balance general". In: DELGADILlO, J. (coord.). Política territorial en México. Hacía un modelo de desarrollo basado en el territorio. México, Secretaría de Desarrollo Social.

Iniciativa que reforma diversas disposiciones de la Ley de Instituto del Fondo Nacional de la Vivienda para los Trabajadores. Gaceta parlamentaria (2009).

INSTITUTO NACIONAL DE ESTADÍSTICA Y GEOGRAFÍA (2000). XII Censo general de población y vivienda. Aguascalientes/México, Autor.

(2005). Sistema urbano y localidades [metadatos y mapoteca digital]. México, Autor.

(2005). I/ Conteo de población y vivienda. Aguascalientes/México, Autor.

(2010). XIII Censo general de población y vivienda. Aguascalientes/México, Autor.

KOSAC, D. (2011). “Fragmentación Urbana y Neoliberalismo Global”. In: PRADILLA, E. Ciudades compactas, dispersas, fragmentadas. México, Universidad Autónoma Metropolitana/Miguel Ángel Porrúa.

MARTÍNEZ, S. e MONROY-ORTIZ, R. (2010). La expansión urbana sobre el campo mexicano. La otra cara de la crisis. Estudios Agrarios, n. 43.

MONROY ORTIZ, R. (2013). Los sistemas urbanos de cuenca en México. Transitando a estrategias integrales de gestión hídrica. Economía, Sociedad y Territorio. El colegio Mexiquense, n. 41.

MONROY-ORTIZ, R. e MONROY, R. (2007). "Saber la biodiversidad para lo urbano. Indicadores básicos”. In: HERNÁNDEZ, E. (coord.). Escenarios de gestión del espacio urbano y regional en México. México, Universidad Autónoma del Estado de México.

ORGANISATION FOR ECONOMIC CO-OPERATION AND DEVELOPMENT (2006). Competitive cities in the global economy. Paris/França, Autor.

PADILLA, S. e RIBBECK, E. (2009). “Colonias populares en la ciudad de México. Urbanismo informal y autoconstrucción”. In: PADILLA, S. (comp.). Urbanismo Informal. México, Universidad Autónoma Metropolitana.

POGGIESE, H. (2004). “Alianzas transversales, reconfiguración de la política y desarrollo urbano: escenarios del presente y del futuro". In: TORRES, A. (coord.). El rostro urbano de América Latina. Buenos Aires, Consejo Latinoamericano de Ciencias Sociales.

POLESE, M. (1994). Economía urbana y regional. Cartago, Libro Universitario regional.

PRADILLA, E. (2008). "La globalización imperialista y las ciudades latinoamericanas". In: RAMIREZ, B. (coord.). Formas territoriales. Visiones y perspectivas desde la teoría. México, Universidad Autónoma Metropolitana.

PROGRAMA DE NACIONES UNIDAS PARA EL DESARROLLO (2007). Informe sobre Desarrollo Humano. México 2006-2007. Migración y desarrollo humano. México, Autor.

(2009). Informe sobre Desarrollo Humano 2009. Superando barreras: movilidad y desarrollos humanos. Nova York, Autor. 
SECRETARÍA DE DESARROLLO SOCIAL, CONSEJO NACIONAL DE POBLACIÓN, INSTITUTO NACIONAL DE ESTADÍSTICA Y GEOGRAFÍA (2007). Delimitación de las zonas metropolitanas de México 2005. México, Autor.

TALAVERA, L. F.; MORALES, E. e MUÑOZ, F. (2010). El slum mexicano II. Investigación socioeconómica para definir un perfil de la pobreza en México. México, Universidad Nacional Autónoma de México.

TELLO, C. (2010). Sobre la desigualdad en México. México, Universidad Nacional Autónoma de México.

THE WORLD BANK (2009). World development indicators 2009. Washington, Autor.

UNITED NATIONS HUMAN SETTLEMENTS PROGRAMME (2005). Financing urban shelter. Global report on human settlements 2005. Nairobi/Kenya, Autor.

(2006). State of worl's cities 2006/7. The millennium developments goals and urban sustainability: 30 years of shaping the habitat agenda. Nairobi/Kenya, Autor.

VEIGA, D. (2009). “Desigualdades sociales y fragmentación urbana”. In: POGGIESE, H. e COHEN, T. (coords.). Otro desarrollo urbano. Ciudad incluyente, justicia social y gestión democrática. Buenos Aires, Consejo Latinoamericano de Ciencias Sociales.

Texto recebido em 13/jan/2014

Texto aprovado em 16/out/2014 